\title{
Acquired Hepatocerebral Degeneration in the Absence of Portosystemic Shunting
}

\author{
Giovanni Caranci, ${ }^{\mathrm{a}, \mathrm{b}}$ \\ Christian Cordano ${ }^{\circ}$ \\ Edoardo Gianninid $^{d}$ \\ Fabio Bandini ${ }^{\mathrm{a}}$ \\ ${ }^{a}$ Department of Neurology, \\ San Paolo Hospital, Savona, Italy \\ 'Department of Neurology, \\ Sant'Anna e San Sebastiano Hospital, \\ Caserta, Italy \\ 'Multiple Sclerosis Center, \\ Department of Neurology, \\ University of California San Francisco \\ (UCSF), San Francisco, CA, USA \\ 'Department of Gastroenterology, \\ University of Genova, \\ Policlinico San Martino, Genova, Italy
}

Received May 23, 2018

Revised August 11, 2018

Accepted August 14, 2018

\section{Correspondence}

Christian Cordano, $\mathrm{MD}, \mathrm{PhD}$

Multiple Sclerosis Center,

Department of Neurology,

University of California San Francisco

(UCSF), Room NS240,

675 Nelson Rising Ln, San Francisco,

CA 94158, USA

Tel +1-415-502-7777

Fax +1-415-502-7777

E-mail cordanochristian@gmail.com
Dear Editor,

Acquired hepatocerebral degeneration (AHD) is a neurological disorder caused by chronic liver diseases associated with portosystemic shunting. It manifests clinically with cognitive impairment and movement disorders. T1-weighted magnetic resonance imaging (MRI) typically shows bilateral pallidal hyperintensities. ${ }^{1}$

A 57-year-old hypertensive man was admitted with a 2-month history of subacute and progressive parkinsonian syndrome. He reported an alcohol intake of 40 grams per day. The findings of a general physical examination were normal. A neurological examination showed facial hypomimia, global bradykinesia, symmetrical extrapyramidal rigidity with bilateral hand resting tremor, and slowed finger-tap movements; his motor score on part 3 of the Unified Parkinson's Disease Rating Scale (UPDRS) was 32/108. Neuropsychological tests disclosed attention deficit, constructive apraxia, and a Mini Mental State Examination score of 23/30. Laboratory tests showed very mild hyperbilirubinemia $(1.3 \mathrm{mg} / \mathrm{dL})$, hypoalbuminemia $(3.4 \mathrm{~g} / \mathrm{dL})$, and thrombocytopenia $\left(140 \times 10^{3} / \mu \mathrm{L}\right)$. Serum ammonia levels were tested twice, with conflicting results: initially $93 \mu \mathrm{mol} / \mathrm{L}$, and $28 \mu \mathrm{mol} / \mathrm{L} 2$ days later. The prothrombin-time international normalized ratio was 1.32 . Serum copper, ceruloplasmin, and urinary copper excretion were all within the normal ranges. Serum gamma glutamyl transpeptidase was $213 \mathrm{U} / \mathrm{L}$ (normal range $=3-50 \mathrm{U} / \mathrm{L}$ ), glutamate oxaloacetate transaminase was $67 \mathrm{U} / \mathrm{L}$ (normal range $=1-31 \mathrm{U} / \mathrm{L}$ ) and glutamate pyruvate transaminase $53 \mathrm{U} / \mathrm{L}$ (normal range $=1-31$ U/L). Liver elastography showed stiffness compatible with parenchymal fibrosis. A slit-lamp examination did not reveal any Kayser-Fleischer rings. An abdominal ultrasound scan showed evidence of chronic parenchymal liver disease and a normal portal vein diameter $(11.5 \mathrm{~mm})$, with the absence of splenomegaly, collateral splenorenal hematic circles, and umbilical vein recanalization. Ultrasound portal flowmetry disclosed a regular hepatopetal flow. Gastroscopy findings were normal.

A diagnosis of alcoholic hepatic cirrhosis was made, with a Child-Pugh classification of A6. Brain MRI showed a slight bilateral globus pallidus hyperintensity in T1-weighted axial images and a venous developmental abnormality in the left frontal lobe as a collateral finding (Fig. 1). The patient was started on levodopa therapy ( $150 \mathrm{mg} /$ daily), and alcohol intake was stopped. The findings of laboratory examinations 6 months later were almost normal, and the patient's neurological status had greatly improved (UPDRS part 3 score=6/108). Levodopa therapy was continued. The patient refused a second MRI scan.

The present case is unusual due to the presentation of AHD as a consequence of chronic liver disease without clinical or ultrasonographic evidence of a portosystemic shunt. To our knowledge, no similar cases have been reported in the literature. We are aware that ultrasound is not the gold-standard test for identifying a portosystemic shunt. However, we feel confident that the results from the ultrasound scans and portal flowmetry in our patient were sufficiently reliable to represent no evidence of shunting. The pathogenesis of AHD in patients with-

(@) This is an Open Access article distributed under the terms of the Creative Commons Attribution Non-Commercial License (https://creativecommons.org/licenses/by-nc/4.0) which permits unrestricted non-commercial use, distribution, and reproduction in any medium, provided the original work is properly cited. 


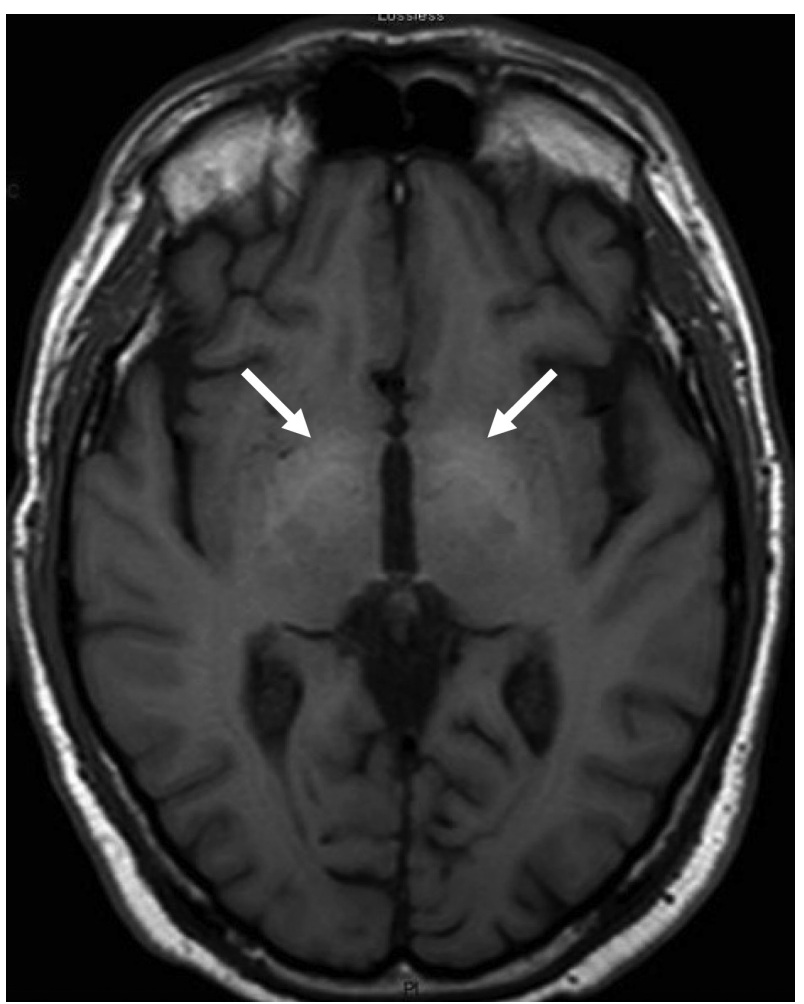

Fig. 1. Moderate bilateral pallidus hyperintensities (arrows) in a T1weighted MRI image.

out clear evidence of portosystemic shunting remains unclear, since portosystemic shunting seems to play a crucial role in the development of AHD. This allows the toxins ammonia, gamma aminobutyric acid, and aromatic amino acids to bypass the first-pass elimination by the liver and thereby accumulate in the brain. ${ }^{1}$ As a consequence, the level of ammonium and the conversion of glutamate into glutamine increase in astrocytes, resulting in cell toxicity and an increased osmotic load with water accumulation. ${ }^{2}$ Manganese seems to be particularly involved in the onset of AHD, with convincing evidence that any pallidal MRI T1-weighted hyperintensities are due to manganese accumulation. ${ }^{1}$ Manganese is a neurotoxic heavy metal that may induce glutamate-mediated excitotoxic injury with swelling of basal ganglia glial cells, cause oxidative stress, and impair dopaminergic neurotransmission by altering postsynaptic $\mathrm{D} 2$ receptors. ${ }^{3}$

To explain why reversible AHD developed in the absence of a clear evidence of portosystemic shunt in our patient, we conjecture that pallidal manganese deposition started before the portosystemic shunt became clinically and radiologically evident. This is consistent with experimental animal models showing pallidal manganese accumulation in rats with chronic liver disease without a portosystemic shunt. ${ }^{4}$ The pallidal manganese content was found to increase not only in portacaval-shunted rats but also in cirrhotic rats without a portosystemic shunt, underlying the possibility that liver failure alone can lead to manganese accumulation in the brain. ${ }^{4}$ Evidence for AHD developing in the absence of portosystemic shunt comes also from the patient reported by Aggarval et al., ${ }^{5}$ who presented with reversible AHD during acute liver failure in the absence of cirrhosis.

This case reports indicates that neurologists should consider that AHD can develop in patients with chronic liver disease also in the absence of either clinical or instrumental evidence of portosystemic shunting.

\section{Conflicts of Interest}

The authors have no financial conflicts of interest.

\section{REFERENCES}

1. Ferrara J, Jankovic J. Acquired hepatocerebral degeneration. J Neurol 2009;256:320-332.

2. Butterworth RF. Neuronal cell death in hepatic encephalopathy. Metab Brain Dis 2007;22:309-320.

3. Liu X, Sullivan KA, Madl JE, Legare M, Tjalkens RB. Manganese-induced neurotoxicity: the role of astroglial-derived nitric oxide in striatal interneuron degeneration. Toxicol Sci 2006;91:521-531.

4. Rose C, Butterworth RF, Zayed J, Normandin L, Todd K, Michalak A, et al. Manganese deposition in basal ganglia structures results from both portal-systemic shunting and liver dysfunction. Gastroenterology 1999;117:640-644.

5. Aggarwal A, Vaidya S, Shah S, Singh J, Desai S, Bhatt M. Reversible Parkinsonism and T1W pallidal hyperintensities in acute liver failure. Mov Disord 2006;21:1986-1990. 